\title{
SOME MULTIDIMENSIONAL OPIAL TYPE INEQUALITIES
}

\section{SONGTING YIN}

Abstract. In this note, we establish some multidimensional Opial type inequalities, which are generalized from the one-dimensional case. Based on calculus and some fundamental inequalities, we first present an elementary proof in Euclidean spaces. Then by using the property of the Minkowski gradient and the so called adapted frame field, we further extend the multidimensional Opial type inequalities to a Minkowski space.

Mathematics subject classification (2020): 26D10, 35A23.

Keywords and phrases: Multidimensional Opial inequality, Euclidean space, Minkowski space.

\section{REFERENCES}

[1] D. BAO, S. S. Chern And Z. M. Shen, An Introduction to Riemann-Finsler Geometry, SpringerVerlag, 2000.

[2] X. G. HE, A short proof of a generalization on Opial's inequality, J. Math. Anal. Appl., 1994, 182 (1): 299-300.

[3] C. OLECH, A simple proof of a certain result of Z. Opial, Ann. Polon. Math., 1960, 8: 61-63.

[4] Z. OpIal, Sur une inégalité, Ann. Polon. Math., 1960, 8: 29-32.

[5] B. G. PachPatte, On multidimensional Opial-type inequalities, J. Math. Anal. Appl., 1987, 126 (1): $85-89$.

[6] Z. M. SHEn, Lectures on Finsler geometry, World Scientific Publishing Co., Singapore, 2001.

[7] G. S. YAng, On a certain result of Z. Opial, Proc. Japan Acad. 1966, 42: 78-83.

[8] C. J. ZhaO, On Opial's type integral inequalities, Mathematics 2019, 7, 375. 